\title{
Towards an Information-Theoretic Approach to Population Structure
}

\author{
Omri Tal ${ }^{1}$ \\ ${ }^{1}$ School of Philosophy and The Cohn Institute for the History and Philosophy of Science \\ and Ideas, Tel Aviv University, Tel Aviv 69978, Israel. \\ talomriepost.tau.ac.il
}

\begin{abstract}
This paper uses an information-theoretic perspective to propose multi-locus informativeness measures for ancestry inference. These measures describe the potential for correct classification of unknown individuals to their source populations, given genetic data on population structure. Motivated by Shannon's axiomatic approach in deriving a unique information measure for communication (Shannon 1948), we first identify a set of intuitively justifiable criteria that any such quantitative information measure should satisfy, and then select measures that comply with these criteria. It is shown that standard information-theoretic measures such as multidimensional mutual information cannot completely account for informativeness when source populations differ in size, necessitating a decision-theoretic approach.
\end{abstract}

\section{Introduction}

Information is the resolution of uncertainty.

-- Claude Shannon

The research on the genetic structure of human populations takes diverse paths and involves complex statistical learning and analysis methods. Two of the most powerful approaches are clustering, which attempts to infer the underlying population structure, and classification, which assigns data of unknown origin to a most probable group. A third category of analysis is high dimensional structural analysis, such as principal component analysis, used for constructing low dimensional qualitative representations. Another common approach is to use diversity, differentiation and distance measures to quantify population relatedness and individual variability. The various links between genetic structure and principles from classical information theory have been pointed out from a variety of perspectives: examining the causal, semantic and transmission sense of information embedded in DNA (Maynard Smith 2000; Godfrey-Smith 2006; Bergstrom and Rosvall 2011), using information-theoretic terms to quantitatively model processes such as drift, mutation, selection, gene flow (Smith 2011), or modeling the evolution of complex traits (Plotkin and Nowak 2000).

The use of an information-theoretical approach to derive measures for the information content of genetic markers has been utilized for assignment of genotypes to their source populations (Rosenberg, $\mathrm{Li}$, et al. 2003) and measuring the informativeness of a marker for relationship or relatedness inference (Wang 2006). Previous work on classification performance and informativeness for assignment in the context of genetic data had identified three basic properties of related measures: [a] higher informativeness with additional loci, [b] higher informativeness with wider population 
divergence, and [c] higher informativeness with larger population sample size (Estoup and Angers 1998; Cornuet, et al. 1999; Edwards 2003; Rosenberg, Li, et al. 2003; Rosenberg 2005; Witherspoon, et al. 2007; Tal 2012). A central goal of this paper is to extend this set of properties, revealing important intrinsic aspects of such information measures. For simplicity, we consider a haploid population model with known allele frequencies from biallelic loci from two subpopulations with given class priors. The approach is motivated by Shannon's axiomatic program in deriving a measure for of the rate of information produced by a discrete information source, formally resembling Entropy in statistical mechanics (Shannon 1948). We first propose a set of necessary and sufficient justifiable properties that any quantitative information measure should satisfy. The next step is to examine a host of candidate measures based on popular divergence measures, distance metrics and classification schemes against these criteria, to finally arrive at a measure that strictly complies with the complete set.

\subsection{The Motivation from Shannon}

In Shannon's groundbreaking 1948 paper, each message received represents gain in information and decrease in uncertainty. Information and uncertainty are thus two sides of the same coin: the more uncertainty there is, the more information we gain by removing the uncertainty (Frigg and Werndl 2011). Shannon posits a measure defined on discrete probabilities of events from an information source:

Can we define a quantity which will measure, in some sense, how much information is "produced" by such a process [a discrete information source], or better, at what rate information is produced? Suppose we have a set of possible events whose probabilities of occurrence are $p_{1}, p_{2}, \ldots, p_{n}$. These probabilities are known but that is all we know concerning which event will occur. Can we find a measure of how much "choice" is involved in the selection of the event or of how uncertain we are of the outcome?

Shannon proceeds to propose a set of three "reasonable" properties any such measure $H$ should comply with: [1] $H$ should be continuous in the $p_{i}$; [2] If all the $p_{i}$ are equal, $p_{i}=1 / n$, then $H$ should be a monotonic increasing function of $n$; and [3] $H$ should be the weighted sum of the values of $H$ resulting from splitting the probabilities into successive choices. It is then proved that the only $H$ satisfying the three assumptions is of the form,

$$
H(X)=-k \sum_{i=1}^{n} p_{i} \log p_{i}
$$

The discussion follows with additional three "interesting properties which further substantiate it as a reasonable measure of choice or information", namely [a] $H>=0$ and is zero only if all the $p_{i}$ are 0 , apart from one $p_{i}$ which is 1 ; [b] maximum value of $H$ is $\log (n)$, attained when all $p_{i}=1 / n$; and [c] any change toward equalization of the probabilities $p_{i}$ increases $H$. Shannon found that only three properties [1-3] were required for arriving at a unique formulation for $H$, whereas these latter properties [a-c] only substantiate $H$ as a reasonable measure. Similarly, we first impose a set of reasonable criteria on any informativeness measure, and justify them from considerations of aspects of classification. Several of these criteria will resemble in structure and motivation Shannon's properties, particularly when expressed symbolically. ${ }^{*}$ Subsequently, we specify a small set of properties that do not have strong prior intuitive justification, but nevertheless provide more insight into the nature of an informativeness measure. In the spirit of Shannon, we specify no optimality

\footnotetext{
* Symbolic forms for the three necessary properties of $H$ were described subsequent to Shannon's paper. Expressed symbolically, they bear a strong resemblance to the formal definition for the criteria in the next section. For instance, $H_{n+1}\left(p_{1}, \ldots, p_{\mathrm{n}}, 0\right)=H_{n}\left(p_{1}, \ldots, p_{\mathrm{n}}, 0\right)$, or $H_{n}\left(p_{1}, \ldots, p_{n}\right) \leq H_{n}(1 / n, \ldots 1 / n)$ or $H_{n}(1 / n, \ldots 1 / n) \leq H_{n+1}(1 /(n+1), \ldots 1 /(n+1))$.
} 
criterion: the numerical value of $H$ is only meaningful in relation to other distributions to which $H$ is applied ( $H$ is unique only up to a constant). ${ }^{\dagger}$

\section{The Criteria}

We denote by $C_{n}$ any information measure across a set of $n$ loci from two haploid populations that complies with a given set of criteria. ${ }^{\ddagger}$ More specifically, $C_{n}(P, Q)$ is a measure of informativeness for classification given a set of $n$ biallelic markers, where $P$ and $Q$ are vectors of known allele frequencies $\left(p_{1}, \ldots, p_{n}\right)$ and $\left(q_{1}, \ldots, q_{n}\right)$ in $\boldsymbol{Q}^{n}$ from populations 1 and 2 respectively, and where $0<p_{i}<1$ and $0<q_{i}<1$. We assume $p_{i}$ and $q_{i}$ are true population parameters of polymorphic loci, i.e., each locus in each population is properly biallelic. Let $\alpha$ be the prior of population 1 (such that $1-\alpha$ is the prior of population 2), the probability that an individual belongs to population 1 when its genotype is unknown (reflecting possible discrepancy in population sizes). In effect, if we denote by $N_{\mathrm{X}}$ the size of population $X$, then $\alpha=N_{1} /\left(N_{1}+N_{2}\right)$. The full notation then becomes $C_{n}(\alpha, P, Q)$ with the shorter notation appearing where contextually sufficient.

[1] Zero: For equal priors $(\alpha=1 / 2), C_{n}=0$ if $n=0$ or $p_{i}=q_{i}$ for all loci $i$. Less formally, for populations of equal size, if there are no loci to examine, or if allele frequencies are exactly equal across loci between populations, there is zero information available for classification. This criterion resembles Shannon's first descriptive property for a condition under which $H=0$.

[2] Non-negativity: $C_{n} \geq 0$. An information measure is expected to be non-negative. This criterion also resembles Shannon's first descriptive property for $H$, namely, $H \geq 0$.

[3] Bound: $C_{n}<1$. The information measure should have an upper bound that signifies the possibility of definite classification. This bound along with non-negativity and the minimum of zero allows $C_{n}$ to be interpreted as a probability or subjective Bayesian certainty about accurate classification. This criterion resembles Shannon's second descriptive property for $H .{ }^{\S}$

[4] Performance: $C_{n}$ should be a monotonic non-decreasing function of $n$. Informally stated, each additional locus may only add information for classification. This criterion resembles Shannon's second requirement for $H$.

[5] Convergence: if allele frequencies at each locus differ between populations by at least $\varepsilon$, where $\varepsilon$ is any predefined value as small as we wish, $0<\varepsilon<1$, then $C_{n}$ asymptotically equals 1 as $\mathrm{n} \rightarrow \infty$. In other words, complete information exists with an infinite number of loci given any minimal predefined frequency difference. ${ }^{* *}$ This criterion implies that in practical sequencing situations, where frequencies of polymorphisms differ by some infinitesimal amount between populations, the inclusion of additional markers eventually results in $C_{n} \rightarrow 1$. Note that criteria \#3 together with \#4 are not equivalent to this criterion.

[6] Neutrality: The inclusion of uninformative loci (for which $q_{i}=p_{i}$ ) should not affect $C_{n}$. Note that this does not imply that an informative locus should necessarily modify $C_{n}$.

[7] Continuity: $C_{n}$ should be continuous in $p_{i}, q_{i}$ and $\alpha$. This criterion resembles Shannon's requirement for continuity in $H$.

\footnotetext{
${ }^{\dagger}$ Subsequent generalizations of entropy, such as Tsallis entropy or Renyi entropy are similarly relative, merely highlighting different aspects of the distribution.

${ }^{\ddagger}$ The use of the term measure with respect to $C_{n}$ should not be interpreted in the strict sense as a mathematical notion of measure over sets.

${ }^{\S}$ If we define $C{ }_{n}^{\prime}=-\log \left(1-C_{n}\right)$ so that it ranges from 0 to infinity like entropy $H$, the measure would lack the benefit of representing maximal informativeness (zero classification error rate). Indeed, $H$ is sometimes normalized to express a "deficiency in entropy" from optimal distribution: Efficiency $=H / \log (n)$.

${ }^{* *}$ Note that criteria \#3 with \#4 are not equivalent to criterion \#5. Without the latter criterion, $C_{n}$ could be less than 1 at the limit.
} 
[8] Dominance: $C_{n} \rightarrow 1$ if $\delta_{i}=\left|q_{i}-p_{i}\right| \rightarrow 1$. A single locus with maximal allele frequency difference should enable definite classification of any individual. ${ }^{\dagger \dagger}$ The asymptotic limit $(\rightarrow 1)$ follows from the Continuity criterion. We note that human SNP data may reveal very high differentiation at many sites, especially at X-linked loci. For e.g., Casto et al. on report 159 X-linked SNPs with frequency delta greater than 0.9 in the Yoruba-Han dataset (Casto, et al. 2010).

[9] Loci invariance: $C_{n}$ should be invariant to different ordering of sequenced loci, i.e., the components of the frequency vectors $P$ and $Q$ may be specified in any order, as long as they remain in sync.

[10] Symmetry invariance: $C_{n}(P, Q)=C_{n}(Q, P) .{ }^{\S \S}$

[11] Allocation invariance: $C_{n}$ should be invariant to the arbitrary choice of the alleles to which we assign the frequency parameters. Thus the concurrent substitution of $p_{i}$ with (1- $\left.p_{i}\right)$ in $P$ and $q_{i}$ with (1$q_{i}$ ) in $Q$ should not affect $C_{n}$.

[12] Priors: $C_{n} \rightarrow 1$ if $\alpha \rightarrow 0$ or $\alpha \rightarrow 1$. At a limit, extremely unequal priors induce complete information for classification; i.e., if one population is infinitely larger than the other, the probability for correct classification is 1 , irrespective of allele frequencies.

In more formal terms:

Let $P=\left(p_{1}, . ., p_{n}\right), Q=\left(q_{1}, \ldots, q_{n}\right)$,

allele frequencies $0<p_{i}, q_{i}<1$, population prior $0<\alpha<1$, and $d_{i}=\left|q_{i}-p_{i}\right|$.

$C_{n}(\alpha, P, Q)$, abbreviated $C_{n}(P, Q)$ or $C_{n}$, should satisfy:

[1] if $\forall i p_{i}=q_{i}$ and $\alpha=\frac{1}{2} \Rightarrow C_{n}=0$

[2] $C_{n} \geq 0$

[3] $C_{n}<1$

[4] $d_{n+1}>0 \Rightarrow C_{n+1}\left(\left(p_{1}, \ldots, p_{n}, p_{n+1}\right),\left(p_{1}, \ldots, q_{n}, q_{n+1}\right)\right) \geq C_{n}(P, Q)$

[5] $\forall \varepsilon>0$, if $\forall i d_{i}>\varepsilon \Rightarrow \lim _{n \rightarrow \infty} C_{n}=1$

[6] $p_{n+1}=q_{n+1} \Rightarrow C_{n+1}=C_{n}$

[7] $C_{n}$ is coninuous in $p_{i}, q_{i}$ and $\alpha$

[8] $\lim _{d_{i} \rightarrow 1} C_{n}=1$

[9] $C_{n}(P, Q)=C_{n}\left(\left(p_{1}, \ldots, p_{i+k}, \ldots, p_{i}, \ldots p_{n}\right),\left(q_{1}, \ldots, q_{i+k}, \ldots, q_{i}, \ldots q_{n}\right)\right)$

[10] $C_{n}(\alpha, P, Q)=C_{n}(1-\alpha, Q, P)$

[11] $C_{n}(P, Q)=C_{n}\left(\left(p_{1}, \ldots, 1-p_{i}, \ldots, p_{n}\right),\left(q_{1}, \ldots, 1-q_{i}, \ldots, q_{n}\right)\right)$

[12] $\lim _{\alpha \rightarrow 0} C_{n}=1$ and $\lim _{\alpha \rightarrow 1} C_{n}=1$

Following Shannon, we suggest a few properties which further substantiate $C_{n}$ as a reasonable measure of information for assignment.

[a] Triviality: At equal priors, $C_{1}=|q-p|$. This formulation for $C_{1}$ reflects the simplest measure for divergence at a single locus: absolute allele frequency difference $(\delta=|q-p|)$.

[b] Population subadditivity: $C_{n}$ complies with a triangle inequality when interpreted as a distance measure between populations. Formally,

$$
C_{n}(\alpha, P, Q)+C_{n}(\beta, Q, R) \geq C_{n}(\gamma, P, R)
$$

\footnotetext{
${ }^{\dagger}$ With $k$ alleles and $c$ populations, we can have Dominance if $k \geq c$.

However, distinctive alleles present in all individuals of one region but absent from individuals outside the region do not exist for human microsatellite data (Rosenberg 2005).

${ }^{\S}$ Nevertheless, exchanging only some of the $p_{i}$ in $P$ with the corresponding $q_{i}$ in $Q$ may change $C_{n}$.
} 
where the three priors are naturally defined in terms of relative population sizes, $\alpha=N_{1} /\left(N_{1}+N_{2}\right)$, $\beta=N_{2} /\left(N_{2}+N_{3}\right), \gamma=N_{1} /\left(N_{1}+N_{3}\right)$. The first two priors define the third: solving for $N_{1}$ and $N_{3}$ in terms of $\alpha$ and $\beta$,

$$
\gamma=\frac{\alpha \beta}{\alpha \beta+(1-\alpha)(1-\beta)} .
$$

The following section examines several proposals based on standard differentiation, divergence, distance and information measures, illuminating the drawbacks of each and preparing the ground for introducing a proposal satisfying the complete set of criteria.

\section{Proposals for $C_{n}$}

\subsection{Differentiation Measures}

Wright's fixation index $F_{\mathrm{ST}}$ is a classic measure of population differentiation. It is commonly defined in terms of expected heterozygosity (Boca and Rosenberg 2011; Tal 2012). In terms of the underlying allele frequencies at a single locus $i$ from two populations we have,

$$
F_{S T}(i)=\frac{H_{T}-H_{S}}{H_{T}}=\frac{\left(q_{i}-p_{i}\right)^{2}}{\left(p_{i}+q_{i}\right)\left(2-p_{i}-q_{i}\right)}
$$

A multilocus $F_{\text {ST }}$ requires separately averaging the numerator and denominator across loci (Weir 1996) such that our first proposal becomes,

$$
C_{n}=F_{S T}=\frac{\sum_{i=1}^{n}\left(p_{i}-q_{i}\right)^{2}}{\sum_{i=1}^{n}\left(p_{i}+q_{i}\right)\left(2-p_{i}-q_{i}\right)}
$$

However, this proposal fails both the Dominance and the Performance criteria (e.g., it is evident that Performance fails when alleles across loci have the same frequency in each population: as $n$ increases $C_{n}$ remains constant).

\subsection{Information-Theoretic Measures}

A powerful measure of statistical dependency is the mutual information. The use of mutual information at a single-locus has been explored in the context of feature selection (Peng, Long and Ding 2005) and informativeness (Rosenberg, Li, et al. 2003). Let $X=\{0,1\}$ represent source populations 1 and 2 respectively, and let $Y_{i}=\{0,1\}$ represent the allele at our biallelic haploid locus $i$, with $p_{i}=\operatorname{Pr}\left(Y_{i}=1 \mid X=0\right)$ and $q_{i}=\operatorname{Pr}\left(Y_{i}=1 \mid X=1\right)$. Formally, assuming equal class priors,

$$
\left(Y_{i} \mid X=0\right) \sim \operatorname{Bernoulli}\left(p_{i}\right), \quad\left(Y_{i} \mid X=1\right) \sim \operatorname{Bernoulli}\left(q_{i}\right), \quad X \sim \operatorname{Bernoulli}\left(\frac{1}{2}\right)
$$

From basic definitions,

$$
\begin{aligned}
& I\left(X ; Y_{i}\right)=\sum_{x=0}^{1} \sum_{y_{i}=0}^{1} p\left(y_{i}, x\right) \log \frac{p\left(y_{i}, x\right)}{p\left(y_{i}\right) p(x)}= \\
& \quad \frac{1-p_{i}}{2} \log \frac{2\left(1-p_{i}\right)}{2-p_{i}-q_{i}}+\frac{p_{i}}{2} \log \frac{2 p_{i}}{p_{i}+q_{i}}+\frac{1-q_{i}}{2} \log \frac{2\left(1-q_{i}\right)}{2-p_{i}-q_{i}}+\frac{q_{i}}{2} \log \frac{2 q_{i}}{p_{i}+q_{i}}
\end{aligned}
$$

Note that this is identical to $I_{n}$ (Rosenberg, Li, et al. 2003, Eq. 4). Averaging across loci and normalizing (assuming all logarithms are to base $e$ ) to comply with the required bounds for $C_{n}$, 


$$
C_{n}=\frac{1}{\log (2) \cdot n} \sum_{i=1}^{n} I\left(X ; Y_{i}\right)
$$

However, this proposal fails the Performance criterion. Since mutual information can incorporate multivariate random variables, we may quantify the dependency of the source population $(X)$ and the joint distribution alleles across $n$ loci $\left(Y_{i}\right){ }^{* * *}$ From basic definitions of mutual information and conditional probability,

$$
\begin{gathered}
I\left(X ;\left[Y_{1}, \ldots, Y_{n}\right]\right)=\sum_{x=0}^{1} \sum_{y_{i}=0}^{1} \ldots \sum_{y_{n}=0}^{1} p\left(y_{1}, \ldots, y_{n}, x\right) \log \frac{p\left(y_{1}, \ldots, y_{n}, x\right)}{p\left(y_{1}, \ldots, y_{n}\right) p(x)}= \\
=\sum_{y_{i}=0}^{1} \ldots \sum_{y_{n}=0}^{1}\left(p\left(y_{1}, \ldots, y_{n} \mid x=0\right) p(x=0) \log \frac{p\left(y_{1}, \ldots, y_{n} \mid x=0\right)}{p\left(y_{1}, \ldots, y_{n}\right)}+\right. \\
\left.\quad+p\left(y_{1}, \ldots, y_{n} \mid x=1\right) p(x=1) \log \frac{p\left(y_{1}, \ldots, y_{n} \mid x=1\right)}{p\left(y_{1}, \ldots, y_{n}\right)}\right)
\end{gathered}
$$

Assuming linkage equilibrium, the joint multivariate distributions $\left[Y_{1}, \ldots, Y_{n} \mid X\right]$ and $\left[Y_{1}, \ldots, Y_{n}\right]$ may be expressed in terms of the allele frequencies $p\left(y_{i} \mid X\right)$,

$$
p\left(y_{1}, \ldots, y_{n} \mid X\right)=\prod_{i=1}^{n} p\left(y_{i} \mid X\right)
$$

and from the law of total probability,

$$
p\left(y_{1}, \ldots, y_{n}\right)=p\left(y_{1}, \ldots, y_{n} \mid X=0\right) p(X=0)+p\left(y_{1}, \ldots, y_{n} \mid X=1\right) p(X=1)
$$

Finally, we introduce class priors, $P(X=0)=\alpha, P(X=1)=1-\alpha$, such that,

$$
\begin{aligned}
& I\left(X ;\left[Y_{1}, \ldots, Y_{n}\right]\right)= \\
& \sum_{k=0}^{2^{n}-1}\left[\alpha h_{k} \log \frac{h_{k}}{\alpha h_{k}+(1-\alpha) g_{k}}+(1-\alpha) g_{k} \log \frac{g_{k}}{\alpha h_{k}+(1-\alpha) g_{k}}\right]
\end{aligned}
$$

Where (noting that by definition $h_{\mathrm{k}}=g_{\mathrm{k}}=1$ for $n=0$ ),

$$
h_{k}=\prod_{i=1}^{n}\left|1-f_{n}(k, i)-p_{i}\right|, \quad g_{k}=\prod_{i=1}^{n}\left|1-f_{n}(k, i)-q_{i}\right|
$$

and where $f_{n}$ is an indicator function for traversing the $2^{n}$ genotypes of each population, transforming the $n$-multiple sum (1) to a single sum,

$$
\left.f_{n}(k, i)=\left\lfloor\frac{k}{2^{i}}\right\rfloor \bmod 2 \quad \text { (the } i^{\text {th }} \text { bit of } k\right)
$$

If all logarithms are taken to base $e$ then $I\left(X ;\left[Y_{1}, \ldots, Y_{n}\right]\right)$ is bounded above by $\log (2)$, which we use for normalization,

$$
C_{n}=\frac{I\left(X ;\left[Y_{1}, \ldots, Y_{n}\right]\right)}{\log (2)}
$$

This proposal fully satisfies the Performance criterion, even for unequal priors. ${ }^{\dagger \dagger}$ However, this measure only satisfies the Dominance criteria for equal priors. It is sufficient to show by counterexample that Dominance fails at some scenario (illustrated by Fig. 1A). Furthermore, this measure

\footnotetext{
*** A similar use of mutual information by (Peng, Long and Ding 2005) is targeted for feature selection, where the object is to find a feature set $S$ with $m$ features that jointly have the largest dependency on the target class - a theoretical scheme called Max-Dependency.

iोi Interestingly, this $C_{n}$ satisfies a strong version of the Performance criterion: it is monotonic increasing at each extra locus, rather than merely monotonic non-decreasing (also for unequal priors).
} 
also fails the Priors criterion since $C_{n}$ approaches 0 (instead of 1) as the prior approaches the extremes of 0 or 1 (Fig 1B).
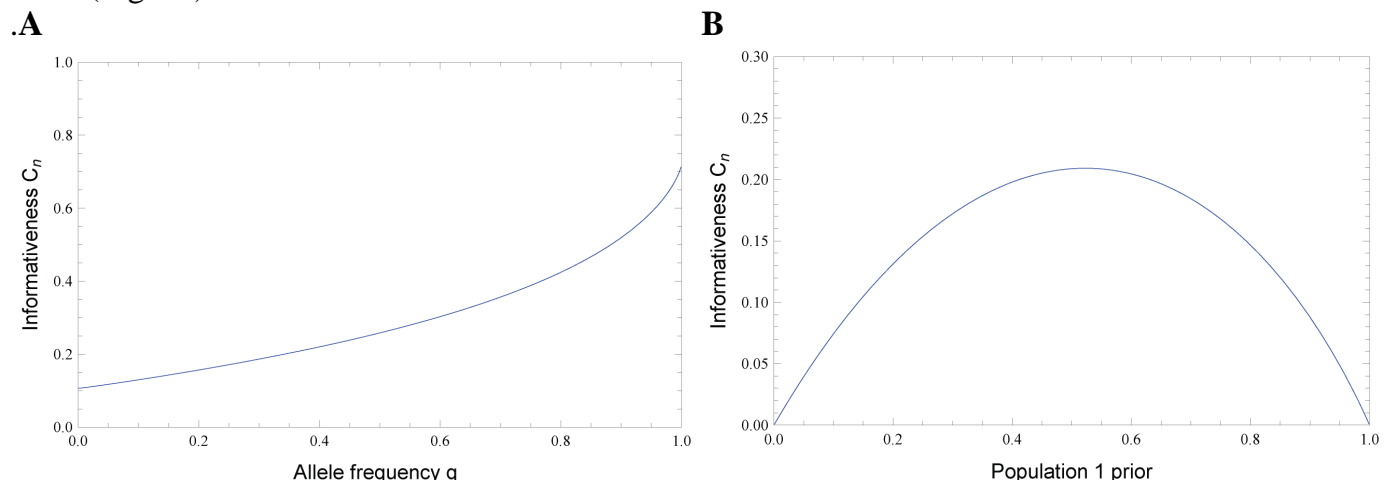

Fig 1. $C_{n}$ based on multilocus mutual information fails two criteria. A: $C_{n}$ does not satisfy the Dominance criterion for unequal population priors. Shown for population 1 prior $\alpha=0.2$, and 4 loci with frequencies $p_{i}=0.1 / q_{i}=0.3$ with the $5^{\text {th }}$ locus reaching full differentiation, $p_{5}=0 / q_{5} \rightarrow 1 . \mid$ B: $C_{n}$ does not satisfy the Priors criterion. Shown for 5 loci with frequencies $p_{i}=0.1 / q_{i}=0.3$ and prior $\alpha$ ranging from 0 to 1 .

\section{$3.3 f$-divergence Measures}

An $f$-divergence is a function $D_{f}(P \| Q)$ that measures the difference between two probability distributions $P$ and $Q$. The divergence is intuitively an average of the odds ratio given by $P$ and $Q$, weighted by the function $f$ that is convex over $(0, \infty)$ and satisfies $f(1)=0$.

$$
D_{f}(P \| Q)=\sum_{x \in \Omega} p(x) f\left(\log \frac{p(x)}{q(x)}\right)
$$

The use of $f$-divergences here is motivated by the properties they hold, which resemble our set of criteria. Such properties are non-negativity (equal to zero if and only if probability densities coincide), convexity, boundedness and various features of invariance (Cichocki and Amari 2010). Moreover, these functions are classically interpreted as modeling discrimination information between hypotheses (Toussaint 1975).

\section{Kullback-Leibler Divergence}

The Kullback-Leibler divergence can be intuitively considered as a distance measure between the two probability densities. For probability distributions $P$ and $Q$ of a discrete random variable their $K L$ divergence (non-negative and unbounded) is defined to be,

$$
D_{K L}(P \| Q)=\sum_{x \in \Omega} p(x) \log \frac{p(x)}{q(x)}
$$

Kullback and Leibler defined a symmetric version of their divergence (Lin 1991),

$$
D_{K L-S}=D_{K L}(P \| Q)+D_{K L}(Q \| P)
$$

The symmetric KL divergence seems to be a good candidate for $C_{n}$. In terms of the genotype probabilities $h$ and $g$ in (3),

$$
D_{K L-S}=\sum_{k=0}^{2^{n}-1}\left[\left(h_{k}-g_{k}\right) \cdot \log \left(\frac{h_{k}}{g_{k}}\right)\right]
$$

Normalization is required to transform its unbounded range of $[0, \infty)$ (Nielsen and Boltz 2011) to $[0,1)$, 


$$
C_{n}=1-\frac{1}{1+D_{K L-S}}
$$

However, this formulation can be shown to fail the Dominance criterion. We also note that it does not have the Population subadditivity property, since the symmetric $K L$ divergence fails the triangle inequality and is therefore not a metric (Khosravifard, Fooladivanda and Gulliver 2007).

\section{Jensen-Shannon Divergence}

A popular symmetrized version of $K L$ divergence is the Jensen-Shannon $(J S)$ divergence, which has some attractive features: it is symmetric, bounded and always defined (even for zero probabilities). A generalized form of the $J S$ Divergence incorporates distribution weights (corresponding to our prior $\alpha$ ),

$$
D_{J S}=\alpha D_{K L}(P \| M)+(1-\alpha) D_{K L}(Q \| M)
$$

where,

$$
M=\alpha P+(1-\alpha) Q
$$

In terms of the genotype probabilities $h$ and $g$ (2.3.0) and setting $m_{k}=\alpha h_{k}+(1-\alpha) g_{k}$,

$$
D_{J S}=\sum_{k=0}^{2^{n}-1}\left[\alpha h_{k} \cdot \log \left(\frac{h_{k}}{\alpha h_{k}+(1-\alpha) g_{k}}\right)+(1-\alpha) g_{k} \cdot \log \left(\frac{g_{k}}{\alpha h_{k}+(1-\alpha) g_{k}}\right)\right]
$$

Noting that the square root of the $J S$ Divergence is a metric (Endres and Schindelin 2003) and using normalization, we produce the following expression,

$$
C_{n}=\sqrt{D_{J S} / \ln (2)}
$$

It is immediately evident that $D_{J S}$ is precisely in the form of the multilocus mutual information in (2). Therefore a formulation of $C_{n}$ based on $D_{J S}$ would also fail the Dominance criterion for unequal priors. An interesting corollary is that if $X \sim \operatorname{Bernoulli}(1-\pi)$,

$$
I\left(X ;\left[Y_{1}, \ldots, Y_{n}\right]\right)=D_{J S}\left(P\left(Y_{1}, \ldots, Y_{n} \mid X=0\right) \| P\left(Y_{1}, \ldots, Y_{n} \mid X=1\right)\right),
$$

\section{The Hellinger Distance}

The Hellinger distance $\left(H_{D}\right)$ is also a type of $f$-divergence and is related to the Bhattacharyya coefficient $\left(B_{C}\right),{ }^{\S \S}$

$$
\begin{aligned}
& H_{D}=\sqrt{1-B_{C}} \\
& B_{C}(P, Q)=\sum_{x \in \Omega} \sqrt{p(x) q(x)}=\sum_{k=0}^{2^{n}-1} \sqrt{h_{k} \cdot g_{k}}
\end{aligned}
$$

Given that $0 \leq H_{D} \leq 1$ (Nielsen and Boltz 2011), we simply let,

$$
C_{n}=H_{D}=\sqrt{1-\sum_{k=0}^{2^{n}-1} \sqrt{h_{k} \cdot g_{k}}}
$$

However, there is no way to introduce the class priors in this formulation: simply replacing the likelihoods $h_{k}$ and $g_{k}$ with the posterior probabilities $\alpha h_{k}$ and $(1-\alpha) g_{k}$ would render the priors arbitrarily interchangeable in the product.

$\$+$ This result was implied in relation to $K L$ Divergence and multiple classes (Vasconcelos and Vasconcelos 2004).

$\S \S \S$ The Bhattacharyya coefficient is closely related to the Bayes error for the special case of equal priors and two classes, and can be used to provide upper and lower bounds for this error (Djouadi, Snorrason and Garber 1990; Aherne, Thacker and Rockett 1998). 


\section{The Mahalanobis Distance}

The Mahalanobis squared distance $\left(\Delta^{2}\right)$ is not strictly an $f$-divergence but a part of a broader class of (Bregman) divergences, and may be seen as an extension of the Euclidean metric,

$$
\Delta^{2}=\left(\mu_{1}-\mu_{2}\right)^{T} \Sigma^{-1}\left(\mu_{1}-\mu_{2}\right) \quad \Sigma=\pi \cdot \Sigma_{1}+(1-\pi) \cdot \Sigma_{2}
$$

The population means, or centroids, are simply the vectors of our allele frequencies (irrespective of possible linkage disequilibrium or the choice of distance metric),

$$
M_{1}=\left(p_{1}, \ldots, p_{n}\right) \quad M_{2}=\left(q_{1}, \ldots, q_{n}\right)
$$

Assuming linkage equilibrium, the diagonal covariance matrix is the mean of the two covariance matrices,

$$
\Sigma=\left(\begin{array}{c}
\alpha p_{1}\left(1-p_{1}\right)+(1-\alpha) q_{1}\left(1-q_{1}\right) \\
\alpha p_{2}\left(1-p_{2}\right)+(1-\alpha) q_{2}\left(1-q_{2}\right) \\
\ldots \ldots \ldots . . \\
\alpha p_{n}\left(1-p_{n}\right)+(1-\alpha) q_{n}\left(1-q_{n}\right)
\end{array}\right)
$$

The distance between the two centroids is therefore,

$$
\Delta\left(M_{1}, M_{2}\right)=\sqrt{\sum_{i=1}^{n} \frac{\left(p_{i}-q_{i}\right)^{2}}{\alpha p_{i}\left(1-p_{i}\right)+(1-\alpha) q_{i}\left(1-q_{i}\right)}}
$$

Alternatively, using an $\mathrm{L}_{1}$ norm $\|x-y\|_{1}=\sum_{i=1}^{n}\left|x_{i}-y_{i}\right|$,

$$
\Delta\left(M_{1}, M_{2}\right)=\sum_{i=1}^{n} \frac{\left|p_{i}-q_{i}\right|}{\sqrt{\alpha p_{i}\left(1-p_{i}\right)+(1-\alpha) q_{i}\left(1-q_{i}\right)}}
$$

Finally, with proper normalization (since the distances are unbounded),

$$
C_{n}=1-\frac{1}{1+\Delta}
$$

This formulation (using either Mahalanobis or the L1-Mahalanobis) satisfies the Dominance criterion also for unequal priors, but fails the Priors criterion, as Fig. 2 demonstrates.

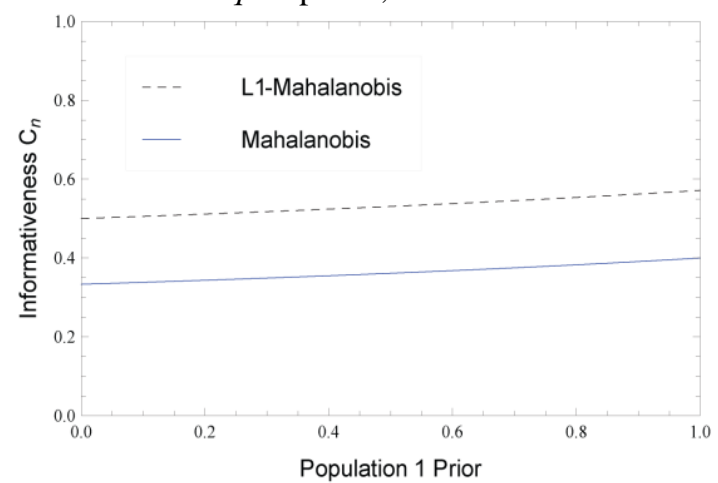

Fig 2. $C_{n}$ based on Mahalanobis distances does not satisfy the Priors criterion (we require $C_{n} \rightarrow 1$ if $\alpha \rightarrow 0$ or $\alpha \rightarrow 1$ ); shown for 4 loci, $p_{i}=0.1 / q_{i}=0.2$ and $\alpha$ ranging from 0 to 1 . 


\subsection{Classifiers}

\section{The Naïve Bayes Classifier}

Quantifying multi-locus information could also be approached from a decision-theoretic perspective, using insights from supervised learning classification schemes. The optimal classifier under known class-conditional densities is the Bayes or maximum-likelihood (ML) classifier, where data are classified according to the most probable class (due to the MAP Rule). The expected error or misclassification rate of the Bayes classifier is called the Bayes error (Hastie, Tibshirani and Friedman 2009). The standard assumption of linkage equilibrium within populations (absence of within-class dependencies) motivates the use of a naïve Bayes classifier, where class-conditional likelihoods are expressed as the product of allele frequencies across the independent loci (Cornuet, et al. 1999; Phillips, et al. 2007). The Bayes error can be formulated as a prior-weighted sum of probabilities over the $2^{n}$ possible genotypes indexed by $k$,

$$
E_{n}=\sum_{k=0}^{2^{n}-1} \min \left(\alpha \cdot h_{k},(1-\alpha) \cdot g_{k}\right)
$$

where the genotype frequencies $g_{k}$ and $h_{k}$ are defined in (3). To gain some intuition consider the expressions for $E_{1}$ and $E_{2}$,

$$
\begin{aligned}
E_{1}= & \min \left(\alpha p_{1},(1-\alpha) q_{1}\right)+\min \left(\alpha\left(1-p_{1}\right),(1-\alpha)\left(1-q_{1}\right)\right) \\
E_{2}= & \min \left(\alpha p_{1} p_{2},(1-\alpha) q_{1} q_{2}\right)+\min \left(\alpha p_{1}\left(1-p_{2}\right),(1-\alpha) q_{1}\left(1-q_{2}\right)\right)+ \\
& +\min \left(\alpha\left(1-p_{1}\right) p_{2},(1-\alpha)\left(1-q_{1}\right) q_{2}\right)+ \\
& +\min \left(\alpha\left(1-p_{1}\right)\left(1-p_{2}\right),(1-\alpha)\left(1-q_{1}\right)\left(1-q_{2}\right)\right)
\end{aligned}
$$

Since for two classes the error rate $E$ of any classifier ranges 0 to $1 / 2$, a straightforward transformation conforming with the Range criterion of $[0,1)$ is $C_{n}=1-2 E$,

$$
C_{n}=1-2 \sum_{k=0}^{2^{n}-1} \min \left(\alpha \cdot h_{k},(1-\alpha) \cdot g_{k}\right)
$$

This is equivalent to the formulation of ORCA (Rosenberg, $\mathrm{Li}$, et al. 2003). An alternative formulation derives from the equivalence of the Bayes error for two classes and the variational distance (a form of $f$-divergence), even for unequal priors (Nguyen, Wainwright and Jordan 2009, section 2.1.1); see Appendix A here for a simple proof given equal priors. ${ }^{* * * *}$ This equivalence results in an alternative expression to (6),

$$
C_{n}=\sum_{k=0}^{2^{n}-1}\left|\alpha \cdot h_{k}-(1-\alpha) \cdot g_{k}\right|
$$

This formulation of $C_{n}$ obeys all 12 criteria and attains the extra properties (Appendix B). Finally, we note that if linkage disequilibrium is nevertheless a feature of the data, the naïve Bayes classifier will have reduced performance, but $C_{n}$ would remain compliant with all criteria and properties. Fig. 3 is a numerical simulation of two criteria.

\footnotetext{
${ }^{* * * *}$ For equal priors it is the only $f$-divergence that is a metric (Khosravifard, Fooladivanda and Gulliver 2007).
} 
A

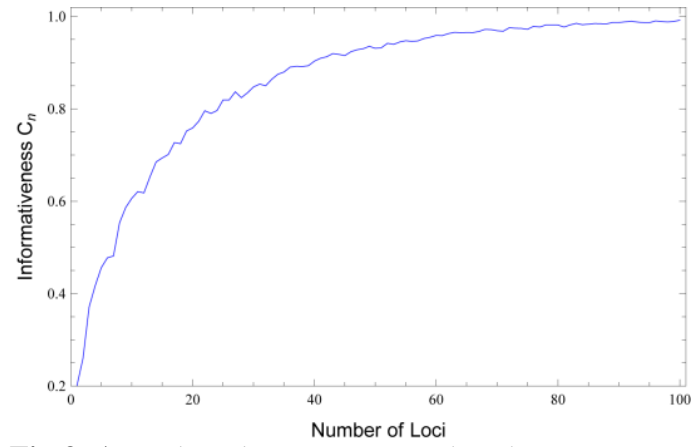

$\mathrm{B}$

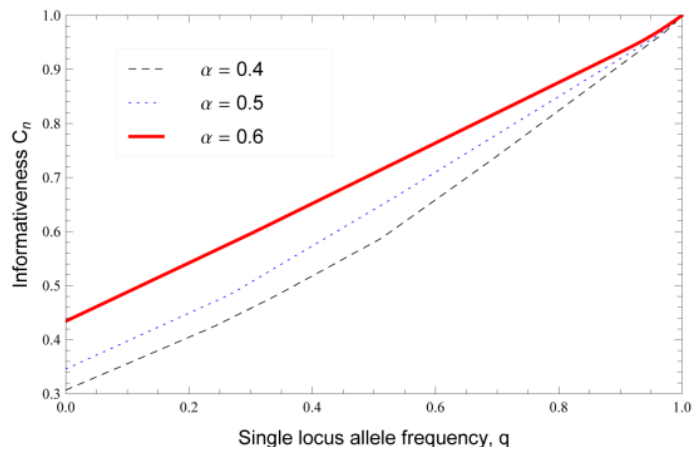

Fig 3. A: $C_{n}$ based on Bayes error has the Convergence property: $C_{n} \rightarrow 1$ as $n \rightarrow \infty$. Shown here for class prior $\alpha=0.4$ and allele frequencies $p_{i}=0.1 / q_{i}=0.3$ using Monte Carlo simulation of (6). | B: $C_{n}$ based on Bayes error has the Dominance property: $C_{n} \rightarrow 1$ as $\left|q_{i}-p_{i}\right| \rightarrow 1$. Shown here for three cases of class priors, under a five loci scenario, where $q_{5}$ changes from $\approx 0$ through $\approx 1$ while $p_{5} \approx 0$; the other 4 loci have frequencies: $p_{1}=0.01 / q_{1}=0.3$, $p_{2}=0.2 / q_{2}=0.35, p_{3}=0.4 / q_{3}=0.3, p_{4}=0.1 / q_{4}=0.15$.

\section{Discussion and Conclusion}

In this paper, we have formulated a measure complying with a predefined set of criteria to capture the informativeness of a collection of markers for population assignment. The informativeness may be seen as the reduction in uncertainty regarding the ancestry of the genotype given these markers. This reduction in uncertainty originates from the fact that each ancestral population has a distinct distribution of over this set of markers (Bercovici and Geiger 2009). We have taken an approach analogous to Shannon's axiomatic program for deriving a measure of the rate of information produced by a discrete information source. Criteria for an information measure are first justified from intuitive considerations, and subsequently employed to arrive at viable formulations. A similar approach was adopted by Lewontin in a widely cited paper on the apportioning of genetic variation in human populations (Lewontin 1972). Lewontin had adopted Shannon's information measure $H$ as a diversity measure for the purpose of ascertaining average population differentiation with respect to a limited set of genetic loci. Four characteristics reminiscent of Shannon's properties for information were specified as a requirement for any (single-locus) diversity measure: [a] minimum when a single allele is present, [b] maximum when all alleles have equal frequencies, [c] generally increase with the number of alleles present, and finally, [d] the pooling of two populations should result in a higher diversity, compared to the average of their separate diversities. In particular, while the first three characteristics are equivalent to three of the properties of $H$ proposed by Shannon, the fourth is original, deemed pertinent specifically to a measure of genetic diversity.

We have shown that a measure of informativeness based on an optimal Bayes classifier complies with the full set of the proposed criteria. However, it is important to emphasize that it cannot be assumed a-priori that any classifier operating on genetic data could similarly be utilized. For e.g., the simple 'population-trait' classifier (Witherspoon, et al. 2007), with an error rate modeled by generalized binomial distributions for haploid populations (Tal 2012), can be shown to fail the Dominance criterion, and in some instances, the Performance criterion. There does not seem to be a simple general heuristic for identifying classifiers that may be utilized for the present purpose; instead, each candidate must be checked against all target criteria. 
Interestingly, compliance with a subset of criteria and one property would deem $C_{n}$ a metric above a set of populations, for equal priors $(\alpha=1 / 2$ is required from criterion \#1). A metric on a set $X$ is a distance function $d: X \times X \rightarrow \mathbf{R}$, where $\mathbf{R}$ is the set of real numbers. If $x, y, z$ are in $X$, this function is required to satisfy four conditions, which express intuitive notions about the concept of distance: ${ }^{\dagger+\dagger \dagger}$

1. $d(x, y) \geq 0$ (our non-negativity criterion) $\$+*$

2. $\quad d(x, y)=0$ if and only if $x=y$ (our zero criterion)

3. $d(x, y)=d(y, x)$ (our symmetry criterion)

4. $\quad d(x, z) \leq d(x, y)+d(y, z)$ (our Population subadditivity property)

Indeed, if we represent populations by their allele frequency vectors, then $C_{n}$ may be seen as a population distance metric. In contrast, standard differentiation measures such as $F_{\mathrm{ST}}$ are often perceived as population distances but are not properly metrics (see (Jardine 1971) for early proposals of dissimilarity coefficients as metrics over a set of populations). ${ }^{\S \S \S \S}$

It was also shown that a previously proposed multi-locus information-theoretic measure based on mutual information $I_{n}$ (Rosenberg, Li, et al. 2003) fails both the Dominance and Priors criteria under unequal population priors (Fig. 1). Therefore, $I_{n}$ could not function as a measure of absolute informativeness; i.e., it could not be used to compare the information content of a collection of loci from one set of populations with a collection of loci from a different set of populations. For instance, consider two populations of unequal size where a different allele is fixed in each at a single locus (i.e., maximum frequency differentiation, $|q-p|=1$ ). If we compute a multi- or single-locus $I_{n}$ that includes this locus, we would have $I_{n}<1$ (since it was shown that Dominance fails for unequal priors), although it is obvious that any proper classification method of unknown genotypes should achieve a $100 \%$ success rate. In contrast, a different pair of populations without complete differentiation at any single locus may have higher $I_{n}$ although it is obvious that there is less information for correct assignment. We note that $I_{n}$ can still function perfectly well for its intended purpose of panel selection (also known as 'feature selection' in statistical learning applications), where different sets of loci (or single markers) from the same meta population are compared for highest informativeness.

Future work will be required to extend the set of criteria and resulting formulations to capture various aspects of practical applications - the use of population samples for estimating allele frequencies and the possible presence of linkage disequilibrium. In addition, it would be useful to generalize the model to comply with diploid genotypes, multiple population scenarios and multiallelic loci.

\section{Appendix}

Appendix A - The equivalence of Bayes error and the variational distance

Here we develop a proof for equal priors only. For the purposes of this proof, $p_{i}$ and $q_{i}$ do not denote allele frequencies but rather genotype frequencies. It is required to prove that,

$$
\sum_{i} \min \left(p_{i}, q_{i}\right)=1-\frac{1}{2} \sum_{i}\left|p_{i}-q_{i}\right|,
$$

where,

\footnotetext{
$\Pi^{\prime \prime \dagger}$ While $C_{n}(P, Q)$ is a metric under equal priors, it is not a norm since the sum $P+Q$ is meaningless.

Note that the upper bound of 1 from criterion \#3 does not detract from the metric quality of $C_{n}$. Take for example the distance metric $d(x, y)=\min \{|x-y|, 1\}$.

$\$ \$ \$$ Another option is to standardize by the number of loci, using $C_{n} / n$, to remove the dependency on $n$.
} 


$$
\sum_{i} p_{i}=1, \sum_{i} q_{i}=1, \quad p_{i}, q_{i}>0, \quad i=2^{k}, k=1,2,3 \ldots
$$

Proof: Let $S$ denote the subset of all indices $i$, for which $p_{i}<q_{i}$ and $T$ denote all other indices $i$. Then for all $p_{i}, q_{i}$ whose indices are in $S$ we have $\left|p_{i}-q_{i}\right|=q_{i}-p_{i}$; similarly, for all $p_{i}$ and $q_{i}$ whose indices are in $T$ we have $\left|p_{i}-q_{i}\right|=p_{i}-q_{i}$. Therefore,

$$
\begin{aligned}
& 2-\sum_{i}\left|p_{i}-q_{i}\right|=\sum_{i} p_{i}+\sum_{i} q_{i}+\sum_{i \in S}\left(p_{i}-q_{i}\right)+\sum_{i \in T}\left(q_{i}-p_{i}\right)= \\
& =\left(\sum_{i \in S} p_{i}+\sum_{i \in T} p_{i}+\sum_{i \in S} q_{i}+\sum_{i \in T} q_{i}\right)+\sum_{i \in S}\left(p_{i}-q_{i}\right)+\sum_{i \in T}\left(q_{i}-p_{i}\right)= \\
& =\left(\sum_{i \in S} p_{i}+\sum_{i \in S} q_{i}+\sum_{i \in S} p_{i}-\sum_{i \in S} q_{i}\right)+\left(\sum_{i \in T} p_{i}+\sum_{i \in T} q_{i}+\sum_{i \in T} q_{i}-\sum_{i \in T} p_{i}\right)= \\
& =2 \sum_{i \in S} p_{i}+2 \sum_{i \in T} q_{i}=2 \sum_{i \in S} \min \left(p_{i}, q_{i}\right)+2 \sum_{i \in T} \min \left(p_{i}, q_{i}\right)=2 \sum_{i} \min \left(p_{i}, q_{i}\right)
\end{aligned}
$$

Thus, $1-\frac{1}{2} \sum_{i}\left|p_{i}-q_{i}\right|=\sum_{i} \min \left(p_{i}, q_{i}\right)$.

Appendix B - Proof of compliance of Bayes $C_{n}$ with all criteria

[1] Zero: If $p_{i}=q_{i}$ for all $i$ then $h_{k}=g_{k}$ for all $k$, and therefore from (7),

$$
C_{n}=|\alpha-(1-\alpha)| \cdot \sum_{k=0}^{2^{n}-1} h_{k}=|\alpha-(1-\alpha)| \cdot 1=|2 \alpha-1|
$$

And if $\alpha=1 / 2$ (equal priors) we have $C_{n}=0$, and also $C_{0}=0$. Note that since $C_{n}(P, P)=|2 \alpha-1|$ it is follows that with unequal priors $C_{n}$ cannot strictly be a metric over vectors of frequencies.

[2] Non-negativity: $C_{n} \geq 0$ follows from the sum of absolute values in (7) being $\geq 0$. A corollary of $\# 1$ above provides the stronger result of $C_{n} \geq|2 \alpha-1|$.

[3] Bound: $C_{n}<1$ follows from the lower bound of zero of the Bayes error and the relation $C_{n}=1$ $2 E_{n}$.

[4] Performance: $C_{n+1} \geq C_{n}$ follows from $E_{n+1} \leq E_{n}$, where $E_{n}$ is the Bayes error given the same distributions (Appendix B.1). An interesting corollary is incorporating an extra locus is not always informative and will not improve classification performance. Formally, without loss of generality,

$$
\forall p_{n+1}, \exists \varepsilon>0, C_{n+1}\left(\left(p_{1}, \ldots, p_{n}, p_{n+1}\right),\left(q_{1}, \ldots, q_{n}, p_{n+1}+\varepsilon\right)\right)=C_{n}(P, Q) .
$$

It can be shown that the range of $\varepsilon$ for which this invariance is satisfied is wider with more unequal priors.

[5] Convergence: First, note that a corollary of criteria \#2 and \#3 is only that $\lim _{n \rightarrow \infty} C_{n} \in[|2 \alpha-1|, 1]$, a weaker result than is demanded by this criterion. ${ }^{* * * * *}$ For the proof that $C_{n} \rightarrow 1$ as $n \rightarrow \infty$ see Appendix B.2.

[6] Neutrality: To prove that $C_{n}=C_{n+1}$ if $p_{n+1}=q_{n+1}$ first note from (5) and (6) that $C_{n+1}$ has twice the number of terms than $C_{n}$. After arranging of terms we have,

$$
C_{n+1}=p_{n+1} C_{n}+\left(1-p_{n+1}\right) C_{n}=C_{n}
$$

[7] Continuity: Since there are no singularities in $C_{n}$ and since $p_{i}=q_{i}$ are real-valued parameters, $C_{n}$ is continuous with respect to its parameters.

\footnotetext{
***** The compliance of $C_{n}$ with this criterion suggests that Theorem 4 in (Rosenberg 2005), which proves for the informativeness measure $I_{n}$ that convergence at infinity is to a number possibly smaller than 1 (for two populations and prior $\alpha$, $\left.\lim _{n \rightarrow \infty} I_{n} \in[\max \{\alpha, 1-\alpha\}, 1]\right)$ is a weaker version of this criterion. Our stronger version applies under practical implementations, where frequencies of polymorphisms analyzed differ by any minimal amount between populations.
} 
[8] Dominance: To see that $C_{n} \rightarrow 1$ if $\left|q_{i}-p_{i}\right| \rightarrow 1$ consider the expression for $E_{n}$ in (4) and without loss of generality examine the effect of $\left|q_{n}-p_{n}\right| \rightarrow 1$. Each of the $2^{n}$ summands is one of either four forms,

$$
\begin{aligned}
& {[a] \min \left\{\alpha p_{1} \cdots p_{n},(1-\alpha) q_{1} \cdots q_{n}\right\}} \\
& {[b] \min \left\{\alpha\left(1-p_{1}\right) \cdots p_{n},(1-\alpha)\left(1-q_{1}\right) \cdots q_{n}\right\}} \\
& {[c] \min \left\{\alpha p_{1} \cdots\left(1-p_{n}\right),(1-\alpha) q_{1} \cdots\left(1-q_{n}\right)\right\}} \\
& {[d] \min \left\{\alpha\left(1-p_{1}\right) \cdots\left(1-p_{n}\right),(1-\alpha)\left(1-q_{1}\right) \cdots\left(1-q_{n}\right)\right\}}
\end{aligned}
$$

Now, from $\left|q_{i}-p_{i}\right| \rightarrow 1$, if $p_{n} \rightarrow 0$ then $q_{n} \rightarrow 1$ and all summands in the form of $a$ and $b$ reduce to zero due to the first term, and all summands in the form of $c$ or $d$ reduce to zero due to the second term. If $p_{n} \rightarrow 1$ then $q_{n} \rightarrow 0$ and all summands in the form of $a$ and $b$ reduce to zero due to the second term, and all summands in the form of $c$ and $d$ reduce to zero due to the first term. This results in $E_{n} \rightarrow 0$ and consequently $C_{n} \rightarrow 1$.

[9] Loci invariance: Since the genotype probabilities $h_{k}$ and $g_{k}$ in (6) are each a commutative product of allele frequencies from all loci, $C_{n}$ is invariant to different ordering of loci.

[10] Symmetry invariance: The truth of $C_{n}(P, Q)=C_{n}(Q, P)$ simply follows from the presence of an absolute value in the formulation of (7).

[11] Allocation invariance: The simultaneous substitution of $p_{i}$ with $\left(1-p_{i}\right)$ and $q_{i}$ with $\left(1-q_{i}\right)$ simply changes the order of the summation terms in (7) and thus does not affect $C_{n}$.

[12] Priors: If $\alpha \rightarrow 0$ or $\alpha \rightarrow 1$ then one of the two terms within the sum in (7) diminishes to zero and what remains in the limit is the sum over all genotype probabilities in one population, which equals 1 , and therefore $C_{n} \rightarrow 1$.

The formulation of $C_{n}$ in terms of the Bayes error also conforms to the two extra properties:

[a] Triviality: In the general case from (7),

$$
C_{1}=\sum_{k=0}^{1}\left|h_{k} \alpha-g_{k}(1-\alpha)\right|=\left|p_{1} \alpha-q_{1}(1-\alpha)\right|+\left|\left(1-p_{1}\right) \alpha-\left(1-q_{1}\right)(1-\alpha)\right|
$$

and for equal priors,

$$
C_{1}=\frac{1}{2}\left|p_{1}-q_{1}\right|+\frac{1}{2}\left|\left(1-p_{1}\right)-\left(1-q_{1}\right)\right|=\left|p_{1}-q_{1}\right|
$$

[b] Population subadditivity: The compliance of $C_{n}$ with the triangle inequality follows from the formulation in (7) which describes the variational distance - a measure that satisfies the triangle inequality, even for unequal priors (Khosravifard, Fooladivanda and Gulliver 2007).

Appendix B.1 - Proof of the Performance criterion for Bayes-based $C_{n}$

We are required to prove that $C_{n+1} \geq C_{n} \forall n \geq 0$ where, $0<p_{i}, q_{i}<1 \quad i=1 \ldots n$.

For simplicity, instead of $C_{n}$ we examine on $E_{n}$, where $C_{n}=1-2 E_{n}$, and would like to prove that $E_{n+1}$ $\leq E_{n}$. From (4),

$$
E_{n}=\sum_{k=0}^{2^{n}-1} \min \left(\alpha \cdot h_{k},(1-\alpha) \cdot g_{k}\right)
$$

In general, the $k$-th summand $X_{k}$ of $E_{n}\left(\right.$ there are $\left.2^{n}\right)$ is split to $X_{2 k}+X_{2 k+1}$ in $E_{n+1}$,

$$
\begin{aligned}
& E_{n}=\ldots+\frac{1}{2} \min \left(\alpha \cdot h_{k},(1-\alpha) \cdot g_{k}\right)+\ldots \\
& E_{n+1}=\ldots+\frac{1}{2} \min \left(\alpha \cdot h_{k} p,(1-\alpha) \cdot g_{k} q\right)+\frac{1}{2} \min \left(\alpha \cdot h_{k}(1-p),(1-\alpha) \cdot g_{k}(1-q)\right)+\ldots
\end{aligned}
$$

where $p, q$ are the frequencies at the $(n+1)$-th locus. There are two cases: 
If $\min \left(\alpha \cdot h_{k},(1-\alpha) \cdot g_{k}\right)=\alpha \cdot h_{k}$, then by simple algebra,

$$
\begin{aligned}
& \min \left(\alpha \cdot h_{k},(1-\alpha) \cdot g_{k}\right)=\alpha \cdot h_{k}=\alpha \cdot h_{k} p+\alpha \cdot h_{k}(1-p) \geq \\
& \min \left(\alpha \cdot h_{k} p,(1-\alpha) \cdot g_{k} q\right)+\min \left(\alpha \cdot h_{k}(1-p),(1-\alpha) \cdot g_{k}(1-q)\right)
\end{aligned}
$$

else, $\min \left(\alpha \cdot h_{k},(1-\alpha) \cdot g_{k}\right)=(1-\alpha) \cdot g_{k}$, and then similarly,

$$
\begin{aligned}
& \min \left(\alpha \cdot h_{k},(1-\alpha) \cdot g_{k}\right)=(1-\alpha) \cdot g_{k}=(1-\alpha) \cdot g_{k} q+(1-\alpha) \cdot g_{k}(1-q) \geq \\
& \min \left(\alpha \cdot h_{k} p,(1-\alpha) \cdot g_{k} q\right)+\min \left(\alpha \cdot h_{k}(1-p),(1-\alpha) \cdot g_{k}(1-q)\right)
\end{aligned}
$$

Finally, summing these inequalities over $k: 1$ to $n$, results in $E_{n+1} \leq E_{n}{ }^{\text {iोit }}$

\section{Appendix B.2 - Proof of the Convergence criterion for Bayes-based $C_{n}$}

We need to prove that for any predefined value $0<\varepsilon<1$, if $\left|q_{i}-p_{i}\right|>\varepsilon$ for all $i$ then $C_{n} \rightarrow 1$. We prove here the equivalent result, $E_{n} \rightarrow 0$, where $C_{n}=1-2 E_{n}$. First, we relate $E_{n}$ to the error rate of the 'population-trait' classifier (Witherspoon, et al. 2007), modeled by generalized binomial distributions (Tal 2012), call it $G_{n}$, by making a small correction of some allele frequencies: the generalized binomial model requires that $p_{\mathrm{i}}<q_{\mathrm{i}}$, so whenever this is not the case replace $p_{i}$ with 1- $p_{i}$ and $q_{i}$ with 1$q_{\mathrm{i}}$, so that $q_{i}-p_{i}>\varepsilon$. This does not change the Bayes error, as can be seen from,

$$
E_{n+1}=\ldots+\frac{1}{2} \min \left(\alpha \cdot h_{k} p,(1-\alpha) \cdot g_{k} q\right)+\frac{1}{2} \min \left(\alpha \cdot h_{k}(1-p),(1-\alpha) \cdot g_{k}(1-q)\right)+\ldots
$$

Now, since the Bayes error is a lower bound on the error achievable from any classifier we have $E_{n} \leq G_{n}$. Next, we relate $G_{n}$ to the misclassification rate from a simple binomial model (Tal, 2012), call it $B_{n}$. For a population with allele frequencies $p_{i}$ the generalized binomial distribution has mean and variance,

$$
\begin{aligned}
& E\left(S_{n}\right)=\sum_{i=1}^{n} E\left(X_{i}\right)=\sum_{i=1}^{n} p_{i}=n \pi \\
& \operatorname{Var}\left(S_{n}\right)=\operatorname{Var}\left(\sum_{i=1}^{n} X_{i}\right)=\sum_{i=1}^{n} p_{i}\left(1-p_{i}\right)=n \pi-n \pi^{2}-\left(\sum_{i=1}^{n} p_{i}^{2}+n \pi^{2}-2 \pi \sum_{i=1}^{n} p_{i}\right)= \\
& =n \pi(1-\pi)-n V_{p}
\end{aligned}
$$

where $\pi$ and $V_{p}$ are the mean and variance of $p_{\mathrm{i}}$, respectively. Since $\operatorname{Var}(X)$ is always lesser or equal to a binomial with $p=\pi$, and since $q_{\mathrm{i}}-p_{\mathrm{i}}>\varepsilon$ for all $i$ implies that $\pi_{q}-\pi_{p}>\varepsilon$, we have that $G_{n} \leq B_{n}$. Next, we show that $B_{n} \rightarrow 0$ as $n \rightarrow \infty$, given $q-p>\varepsilon$.

$$
B_{n}=\sum_{i=0}^{n} \min \left\{\alpha \cdot\left(\begin{array}{l}
n \\
i
\end{array}\right) p^{i}(1-p)^{n-i},(1-\alpha) \cdot\left(\begin{array}{l}
n \\
i
\end{array}\right) q^{i}(1-q)^{n-i}\right\}
$$

Since the minimum of two nonnegative quantities is bounded by their geometric mean, we have,

$$
B_{n} \leq \alpha(1-\alpha) \sum_{i=0}^{n}\left(\begin{array}{l}
n \\
i
\end{array}\right) p^{i / 2} q^{i / 2}(1-p)^{(n-i) / 2}(1-q)^{(n-i) / 2}
$$

We now note that the geometric mean is bounded by the arithmetic mean, with equality iff the two quantities are equal. In particular, $\sqrt{p} \sqrt{q}=r(p+q) / 2$ for some $0<r<1$, and $\sqrt{(1-p)} \sqrt{(1-q)}=s(1-(p+q) / 2)$ for some $0<s<1$. Note we assume $p \neq q$ here. So,

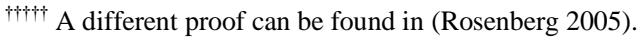




$$
B_{n} \leq \alpha(1-\alpha) \sum_{i=0}^{n}\left({ }_{i}^{n}\right) r^{i} s^{n-i}\left(\frac{p+q}{2}\right)^{i}\left(1-\frac{p+q}{2}\right)^{n-i}
$$

Next, observe that $r^{i} s^{n-i} \leq t^{n}$ where $0<t=\max (r, s)<1$. So we have,

$$
B_{n} \leq \alpha(1-\alpha) t^{n} \sum_{i=0}^{n}\left(\begin{array}{l}
n \\
i
\end{array}\right)\left(\frac{p+q}{2}\right)^{i}\left(1-\frac{p+q}{2}\right)^{n-i}
$$

By the binomial theorem the sum is just 1 . So we get,

$$
B_{n} \leq \alpha(1-\alpha) t^{n}
$$

Since $0<t<1$, we finally get $\lim _{n \rightarrow \infty} B_{n}=0$ as needed.

Finally, from $E_{n} \leq G_{n} \leq B_{n}$ we have $E_{n} \rightarrow 0$ as $n \rightarrow \infty$, and from $C_{n}=1-2 E_{n}$ we get $C_{n} \rightarrow 1$.

\section{Acknowledgements}

The author would like to thank Jonathan Rosenblatt and Tamir Tassa for helpful suggestions on mathematical and computational issues.

\section{References}

Aherne, F., N. Thacker, and P. Rockett. "The Bhattacharyya metric as an absolute similarity measure for frequency coded data." Kybernetika, vol. 34, no. 4, 1998: 363-368.

Bercovici, S., and D. Geiger. "Inferring Ancestries Efficiently in Admixed Populations with Linkage Disequilibrium." Journal of Computational Biology 16:8, 2009.

Bergstrom, C., and M. Rosvall. "The transmission sense of information." Biology and Philosophy 26(2), 2011: 159-176.

Boca, S.M., and N.A. Rosenberg. "Mathematical properties of Fst between admixed populations and their parental source populations." Theoretical Population Biology 80, 2011: 208-216.

Casto, AM, JZ Li, D Absher, R Myers, S Ramachandran, and MW. Feldman. "Characterization of $\mathrm{X}$-Linked SNP genotypic variation in globally distributed human populations." Genome Biology 11:R10, 2010.

Cichocki, A., and S-I. Amari. "Families of Alpha- Beta- and Gamma- Divergences: Flexible and Robust Measures of Similarities." Entropy, 12, 2010: 1532-1568.

Cornuet, J.M., S. Piry, G. Luikart, A. Estoup, and M. Solignac. "New methods employing multilocus genotypes to select or exclude populations as origins of individuals." Genetics 153, 1999.

Djouadi, A., O. Snorrason, and F. Garber. " The quality of Training-Sample estimates of the Bhattacharyya coefficient." IEEE Transactions on Pattern Analysis and Machine Intelligence 12 (1), 1990: 92-97.

Edwards, A.W.F. "Human genetic diversity: Lewontin's fallacy." BioEssays 25, 2003: 798-801.

Endres, D.M., and J. E. Schindelin. "A new metric for probability distributions." IEEE Trans. Inf. Theory, 49, 2003: 1858-60.

Estoup, A., and B. Angers. "Microsatellites and minisatellites for molecular ecology: theoretical and empirical considerations." In Advances in Molecular Ecology, by G. Carvalho, 55-86. NATO press, 1998.

Frigg, R., and C. Werndl. "Entropy - A Guide for the Perplexed." In Probabilities in Physics, by Beisbart \& S. Hartmann, 115-42. Oxford University Press, 2011.

Godfrey-Smith, P. "Information in Biology." In The Cambridge Companion to the Philosophy of Biology, by M Ruse and Hull D. Cambridge University Press, 2006.

Hastie, T., R. Tibshirani, and J.H. Friedman. The Elements of Statistical Learning, 2nd Edition. Springer Verlag, 2009. 
Jardine, N. "Patterns of Differentiation Between Human Local Populations." Philosophical Transactions of the Royal Society of London. Series B, Biological Sciences. Vol. 263, No. 846, 1971: $1-33$.

Khosravifard, M., D. Fooladivanda, and T.A. Gulliver. "Confliction of the convexity and metric properties in f-divergences." IEICE Trans. on Fundamentals E90-A 9, 2007: 1848-1853.

Lewontin, R. "The apportionment of human diversity." Evolutionary Biology, 6, 1972: 381-398.

Lin, J. "Divergence measures based on the shannon entropy." IEEE Transactions on Information Theory 37 (1), 1991: 145-151.

Maynard Smith, J. "The Concept of Information in Biology." Philosophy of Science 67, 2000: 177194.

Nguyen, X., M.J. Wainwright, and M.I. Jordan. "On surrogate loss functions and f-divergences." Annals of Statistics, 37, 2009: 876-904.

Nielsen, F., and S. Boltz. "The Burbea-Rao and Bhattacharyya centroids." IEEE Transactions on Information Theory, arXiv:1004.5049v2, 2011.

Peng, H., F. Long, and C. Ding. "Feature selection based on mutual information criteria of maxdependency, max-relevance, and min-redundancy." IEEE Transactions on Pattern Analysis and Machine Intelligence 27 (8), 2005: 1226-1238.

Phillips, C., et al. "Inferring ancestral origin using a single multiplex assay of ancestry-informative marker SNPs." Forensic Sci Int Genetics 1, 2007: 233-235.

Plotkin, J.B., and M.A. Nowak. "Language evolution and information theory." Journal of Theoretical Biology, 205(1), 2000: 147--159.

Rannala, B., and J. L. Mountain. "Detecting immigration by using multilocus genotypes." Proc. Natl. Acad. Sci. USA 94, 1997: 9197-9221.

Reza, F.M. An Introduction to Information Theory. New York: McGraw-Hill. New York: Dover 2010 Ed., 1961.

Rosenberg. "Algorithms for selecting informative marker panels for population assignment." Journal of Computational Biology 12, 2005: 1183-1201.

Rosenberg, N.A. "A Population-Genetic Perspective on the Similarities and Differences among Worldwide Human Populations." 2011: Human Biology: Vol. 83: Iss. 6.

Rosenberg, N.A., L.M. Li, R. Ward, and J.K. Pritchard. "Informativeness of Genetic Markers for Inference of Ancestry." Am. J. Hum. Genet., 73, 2003: 1402-1422.

Shannon, C.E. "A mathematical theory of communication." The Bell System Technical Journal, 27, 1948: 379-423.

Smith, R.D. "Information Theory and Population Genetics." submitted to PLoS One, 2011.

Tal, O. "The Cumulative Effect of Genetic Markers on Classification Performance: Insights from Simple Models." Journal of Theoretical Biology. Volume 293, 21 January, 2012: 206-218.

Toussaint, G.T. "Sharper lower bounds for discrimination information in terms of variation." IEEE Trans. inform. Theory, vol. IT-21. no. 1, 1975: 99-100.

Vasconcelos, N., and M. Vasconcelos. "Scalable Discriminant Feature Selection for Image Retrieval and Recognition." CVPR (2), 2004: 770-775.

Wang, J. "Informativeness of genetic markers for pairwise relationship and relatedness inference." Theoretical Population Biology 70, 2006: 300-321.

Weir, B.S. Genetic Data Analysis II: Methods for discrete population genetic data (2nd. ed.). Sinauer Assoc., Sunderland, MA., 1996.

Witherspoon, D.J., et al. "Genetic similarities within and between human populations." Genetics 176, 2007: 351-359. 